\title{
ON SYLOW INTERSECTIONS IN FINITE GROUPS
}

\author{
GEOFFREY R. ROBINSON
}

\begin{abstract}
In general, for a given prime $p$ and finite group $G$, there need not be Sylow $p$-subgroups $P$ and $Q$ of $G$ with $P \cap Q=O_{p}(G)$. In this paper we show that if $G$ is $p$-soluble, and $p$ is not 2 or a Mersenne prime, then such Sylow $p$-subgroups exist (also we give conditions guaranteeing the existence of such Sylow subgroups when $p$ is 2 or a Mersenne prime). We also show that if $G$ is not $p$-soluble, but $p$ is odd and the components of $G / O_{p}(G)$ are in a certain class of quasi-simple groups, then there are Sylow $p$-subgroups $P$ and $Q$ of $G$ with $P \cap Q=O_{p}(G)$, unless perhaps $p$ is a Mersenne prime. When $G$ is p-soluble, our work extends results of $\mathrm{N}$. Itô [2].
\end{abstract}

In general, when $G$ is a finite group and $p$ is a prime divisor of $|G|$, there need not be Sylow $p$-subgroups $P$ and $Q$ of $G$ such that $P \cap Q=O_{p}(G)$. The aim of this note is to prove that in many situations, though, there are such Sylow $p$-subgroups.

Our first observation is that "regular-orbit" theorems such as that of Hargreaves [1] can be used to establish the existence of such Sylow subgroups in $p$-solvable groups when $p \neq 2$ or a Mersenne prime. More precisely, we can prove the following theorem, which slightly strengthens a result of Itô [2].

THEOREM 1. Let $G$ be a finite $p$-solvable group, and $P \in \operatorname{Sylp}(G)$. Then there is a Sylow p-subgroup, $Q$, of $G$ with $P \cap Q=O_{p}(G)$, unless, perhaps, one of the following situations occur:

(a) $p=2, P / O_{p}(G)$ involves $Z_{2} \sim Z_{2}$ and $\left|O_{p, p^{\prime}}(G)\right|$ is divisible by the square of $a$ Fermat prime, or the square of a Mersenne prime.

(b) $p$ is a Mersenne prime, $P / O_{p}(G)$ involves $Z_{p} \sim Z_{p}, P / O_{p}(G)$ does not centralize $O_{2}\left(G / O_{p}(G)\right)$, and $(p+1)^{p}$ divides $|G|$.

Proof. We proceed by induction on $|G|$. Suppose that neither (a) nor (b) hold. Certainly we may suppose that $O_{p}(G)=1_{G}$. By the Hall-Higman centralizer lemma, $C_{G}\left(O_{p^{\prime}}(G)\right) \leqslant O_{p^{\prime}}(G)$. Thus $O_{p}\left(P O_{p^{\prime}}(G)\right)=1_{G}$, so we may suppose that $G=$ $P O_{p^{\prime}}(G)$.

We claim that $O_{p^{\prime}}(G)$ is a $q$-group for some prime $q \neq p$. Let $\left\{r_{i}: 1 \leqslant i \leqslant n\right\}$ be the set of prime divisors of $\left|O_{p^{\prime}}(G)\right|$, and for each $i$, let $R_{i}$ be a $P$-invariant Sylow $r_{i}$-subgroup of $O_{p^{\prime}}(G)$. Suppose that $n>1$. If $p$ is not a Mersenne prime, then neither (a) nor (b) hold in $P R_{i}$, so by induction there are some $x_{i}$ in $R_{i}$ with $P \cap P^{x_{i}}=C_{p}\left(R_{i}\right)$. Let $x=x_{1} x_{2} \cdots x_{n}$. Then $x$ centralizes $P \cap P^{x}$. Let $u \in P \cap$ $P^{x}$. Then $x^{u}=x$, so that $x_{1}^{u} x_{2}^{u} \cdots x_{n}^{u}=x_{1} x_{2} \cdots x_{n}$. Now $x_{i}^{u} \in R_{i}$ for each $i$, and 
each element of $O_{p^{\prime}}(G)$ has a unique expression of the form $z_{1} z_{2} \cdots z_{n}$, where each $z_{i} \in R_{i}$. Thus $x_{i}^{u}=x_{i}$ for each $i$, so that $u \in P \cap P^{x_{i}}$ for each $i$. Consequently, $u \in \cap{ }_{i=1}^{n} C_{p}\left(R_{i}\right)=C_{p}\left(O_{p^{\prime}}(G)\right)=1_{G}$. Thus $P \cap P^{x}=1_{G}$, and we are done. Thus $p$ is a Mersenne prime, and for some $i$, (b) must hold within $P R_{i}$ (or we can repeat the above argument).

We may assume then that $r_{1}=2$. For $i>1$, by induction, there is $x_{i} \in R_{i}$ with $P \cap P^{x_{i}}=C_{p}\left(R_{i}\right)$. Let $x=x_{2} \cdots x_{n}$. Then as above, $P \cap P^{x} \leqslant \cap_{i=2}^{n} C_{p}\left(R_{i}\right)$. We may assume then that there is some nonidentity element $u$ in $\cap_{i=2}^{n} C_{p}\left(R_{i}\right)$. Then $O_{p^{\prime}}(G)=R_{1} C_{O_{p^{\prime}}(G)}(u)$, so that $\left[O_{p^{\prime}}(G), u\right] \leqslant\left[R_{1}, u\right] \leqslant R_{1}$, and hence $\left[O_{p^{\prime}}(G), u\right] \leqslant$ $\mathrm{O}_{2}(G)$.

Now $\left[O_{p^{\prime}}(G), u\right] \neq 1_{G}$, so $\left[O_{2}(G), u\right] \neq 1_{G}$, as $\left[O_{p^{\prime}}(G), u\right]=\left[O_{p^{\prime}}(G), u, u\right] \leqslant\left[O_{2}(G)\right.$, $u$ ]. In particular $P$ does not centralize $O_{2}(G)$. Now, however, as (b) does not hold in $G$, (b) does not hold within $P R_{1}$, contrary to assumption.

Hence $n=1$, so that $O_{p^{\prime}}(G)$ is a $q$-group for some prime $q \neq p$. Let $R=O_{p^{\prime}}(G)$. We claim that $R$ is elementary abelian. If not, then $O_{p}(G / \Phi(R))=1_{G}$, and by induction, there is $\bar{x}$ in $\bar{G}=G / \Phi(R)$ with $\bar{P} \cap \bar{P}^{\bar{x}}=1 \bar{G}$. For any preimage $x$ of $\bar{x}$, we have $P \cap P^{x}=1_{G}$, so we may indeed suppose that $R$ is elementary abelian.

Now, as $R$ is elementary abelian, and neither (a) nor (b) hold in $G=P R$, we may appeal to the main theorem of Hargreaves [1] to conclude that for some $y \in R$, $C_{p}(y)=1_{G}$. However, $P \cap P^{y} \leqslant C_{p}(y)$, as $y \in O_{p^{\prime}}(G)$, so that $P \cap P^{y}=1_{G}$.

The proof of Theorem 1 is complete.

REMARK. Of course, conditions (a) and (b) both fail to be satisfied when $G$ has odd order. We also remark that if either (a) or (b) is satisfied, it is possible that $O_{p}(G)$ can fail to be a Sylow intersection, and the simplest such examples are groups of the form $P Q$, where $P$ is a $p$-group faithfully represented as a group of automorphisms of the elementary abelian $q$-group $Q(q$ a prime $\neq p)$, when $P$ fails to have a regular orbit on $Q$.

We next consider a more general class of groups. First, a definition:

Definition. Let $p$ be an odd prime. Let $\delta(p)=$ (nonabelian finite simple groups $H$ such that for $P \in \operatorname{Sylp}(\operatorname{Aut}(H)), P$ has at least two orbits (by conjugation) on involutions $t$ of $H$ with $P \cap P^{t}=1_{H}$ (where $H$ is considered as a normal subgroup of $\operatorname{Aut}(H))$.

REMARK. It seems that most finite simple groups, of order divisible by $p$, are in $\delta(p)$. A proof that all (known) simple groups of order divisible by $p$ are in $\delta(p)$ seems feasible, but would be extremely tedious.

Before we state and prove Theorem 2, we require a technical lemma. Let $p$ be an odd prime.

Lemma 1. Let $G=P E$, where $P \in \operatorname{Sylp}(G)$ and $E$ is a minimal normal subgroup of $G$ which is a direct product of nonabelian simple groups, each of which is in $\mathcal{S}(p)$. Then there are involutions $t$ and $u$ of $G$ (which, of course, lie in $E$ ) such that $P \cap P^{t}=P \cap$ $P^{u}=O_{D}(G)$, and $t$ and $u$ lie in different orbits under conjugation by $P$.

Proof. We proceed by induction on $|G|$. If $E$ is simple, the result follows (upon passage to $\left.G / O_{p}(G)\right)$ by using the definition of $\delta(p)$. Thus we may suppose that $E$ 
is not simple. Let $L$ be a simple normal subgroup of $E$, and let $S=N_{p}(L)$. Let $M$ be a maximal subgroup of $P$ containing $S$. (Note that $S \neq P$ as $E$ is minimal normal in $E P$, but $E$ is not simple.)

Then for some $x \in P \backslash M, E=Y Y^{x} Y^{x^{2}} \cdots Y^{x^{p}}$ ' (where this latter product is direct), where $Y=L^{M}$ (a direct product of $[M: S]$ isomorphic copies of $L$ ).

Let $a$ and $b$ be involutions of $Y$ in different $M$-orbits such that $M \cap M^{a}=M \cap$ $M^{b}=O_{p}(M Y)=C_{M}(Y)$ (by induction, such involutions exist). Let $z=a b^{x} b^{x^{2}} \ldots$ $b^{x^{p-1}}$ and let $w=b a^{x} a^{x^{2}} \cdots a^{x^{p-1}}$. Then $z$ and $w$ are involutions of $E$. They are not conjugate under the action of $P$, for if $z^{x^{\prime} m}=w$, where $0 \leqslant i \leqslant p-1$, and $m \in M$, then if $i>1, b^{x^{p+1} m}=a^{x}$, so $b^{x^{p\left(x m x^{-1}\right)}}=a$, a contradiction as $x^{p} \in M$, but $a$ and $b$ are not conjugate under the action of $M$. If $i \leqslant 1$, we obtain $b^{x^{1+1} m}=a^{x^{1+1}}$, again a contradiction.

It remains only to prove that $P \cap P^{z}=P \cap P^{w}=O_{p}(G)$. We prove that $P \cap P^{z}$ $=O_{p}(G)$ for interchanging the roles of $a$ and $b$ then establishes that $P \cap P^{w}=$ $O_{p}(G)$.

Suppose that $u \in P \cap P^{z}$. Then $u^{-1} u^{z} \in P$, so that $z z^{u} \in P \cap E$. Let $u=x^{i} m$, where $0 \leqslant i \leqslant p-1$, and $m \in M$. Then

$$
\left(a b^{x} b^{x^{2}} \cdots b^{x^{p-1}}\right)\left(a^{x^{\prime} m} b^{x^{\prime+1} m} \cdots\right) \in P \cap E .
$$

We first note that $i=0$. If not, then $b^{x^{\prime}} a^{x^{\prime} m} \in P \cap Y^{x^{\prime}}$ so $b a^{x^{\prime} m x^{\prime \prime}} \in P \cap Y=M$ $\cap Y$, which forces $b$ and $a^{x^{\prime} m x^{-1}}$ to be conjugate by an element of $M$ (as $a$ and $b$ are involutions), a contradiction. Thus $i=0$.

Hence $\left(a b^{x} \cdots b^{x^{p-1}}\right)\left(a^{m} b^{x m} \cdots b^{x^{p-1} m}\right) \in P \cap E$, so for $1 \leqslant i \leqslant p-1, b^{x^{i}} b^{x^{\prime} m}$ $\in P \cap Y^{x^{\prime}}$, so $b b^{x^{\prime} m x^{-1}} \in M \cap Y$. Thus $x^{i} m x^{-i} \in M \cap M^{b}=C_{M}(Y)$. Also, $a a^{m} \in$ $M \cap Y$, so $m \in M \cap M^{a}$ and $m \in C_{M}(Y)$. Hence $m \in \cap_{i=0}^{p-1} C_{M}\left(Y^{x^{i}}\right)=C_{p}(E)=$ $O_{p}(G)$. Thus we have shown that $P \cap P^{z}=O_{p}(G)$, as claimed.

Theorem 2. Let $G$ be a finite group and $p$ be an odd prime. Let $P \in \operatorname{Sylp}(G)$. Suppose that $O_{p}(G)=1_{G}$, and if $p$ is a Mersenne prime, assume either that $O_{2}(G) \leqslant$ $Z(G)$, or else that $P$ does not involve $Z_{p} \sim Z_{p}$. Suppose further that for each component, $L$, of $G$ such that $p|| L \mid, L / Z(L)$ is in $\mathcal{S}(p)$. Then there is a Sylow p-subgroup, $Q$, of $G$ such that $P \cap Q=1_{G}$.

Proof. We proceed by induction on $|G|$. Since $C_{G}\left(F^{*}(G)\right) \leqslant F^{*}(G)$ and $O_{p}(G)=$ $1_{G}$, we see that $O_{p}\left(F^{*}(G) P\right)=1_{G}$. We may suppose, then, that $G=P F^{*}(G)$. If $O_{2}(G) \leqslant Z(G)$, we can suppose that $F^{*}(G)=E(G) O(F(G))$.

Next, we claim that $Z(E(G))=1_{G}$. For let $Z=Z(E(G))$. If $Z \neq 1_{G}$, then by induction, for some $\bar{x} \in \bar{G}(=G / Z)$, we have $\bar{P} \cap \bar{P}^{\bar{x}}=1_{\bar{G}}$ (for it is easy to check that $\left.O_{p}(\bar{G})=1 \bar{G}\right)$. Then for some preimage, $x$ say, of $\bar{x}$, we have $P \cap P^{x}=1_{G}$.

Now we claim that $F(G)=1_{G}$. If $F(G) \neq 1_{G}$, let $F=F(G)$ and $E=E(G)$. Since $Z(E)=1_{G}, F^{*}(G)=F \times E$. By Theorem 1 , there is some $f \in F$ such that $P \cap P^{f}$ $=O_{p}(P F)=C_{p}(F)$. By induction, there is some $e \in E$ such that $P \cap P^{e}=C_{p}(E)$. It is easy to check that $P \cap P^{e f} \subseteq C_{p}(E F)=1_{G}$. Thus $F=1$. A similar argument, using Theorem 1 , shows that $O_{p^{\prime}}(G)=1_{G}$. 
If $E$ is not a minimal normal subgroup, but $E=E_{1} \times E_{2}$, where each $E_{i} \triangleleft E P$, then, by induction for each $i$ there is an $e_{i} \in E_{i}$ with $P \cap P^{e_{1}}=C_{p}\left(E_{i}\right)$. It quickly follows that $P \cap P^{e_{1} e_{2}}=1_{G}$. Thus $E$ is a minimal normal subgroup of $G$. Now we may appeal to Lemma 1 to conclude that for some $e \in E$, we have $P \cap P^{e}=1_{G}$. The proof of Theorem 2 is complete.

\section{REFERENCES}

1. B. B. Hargreaves, The existence of regular orbits for nilpotent groups, J. Algebra 72 (1981), 54-100.

2. N. Itô, Über den Kleinsten p-Durchschnitt ausflösbarer Gruppen, Arch. Math. (Basel) 9 (1958), $27-32$.

Department of Mathematics, University of Chicago, Chicago, Illinois 60637 